\title{
Bouveret syndrome: A series of cases that illustrates a rare complication of chronic cholelithiasis
}

\author{
Brianne Runyan ${ }^{1}$, Michael L. Caparelli ${ }^{1}$, Jason Batey ${ }^{1}$, Shyam Allamaneni ${ }^{1}$, and Steven Perlman ${ }^{2}$ \\ Departments of ${ }^{1}$ General Surgery and ${ }^{2}$ Radiology, The Jewish Hospital of Cincinnati, Cincinnati, OH, USA
}

\begin{abstract}
Bouveret syndrome is defined as gastric outlet obstruction secondary to the impaction of a large gallstone in the proximal gastrointestinal tract. The obstruction occurs as result of a bilio-enteric or bilio-gastric fistula. This clinical entity is a rare variant of the more commonly recognized gallstone ileus, which tends to cause small bowel obstruction of the terminal ileum. The typical presentation of Bouveret syndrome consists of nausea, vomiting and abdominal pain secondary to obstruction. Diagnosis often requires radiographic imaging with computed tomography, which typically shows pneumobilia or a cholecystoduodenal fistula. Herein is a series consisting of three cases of Bouveret syndrome involving a bilioenteric, cholecystoduodenal, and choledochoduodenal fistula, respectfully, all of which required operative management. A discussion of the current literature regarding management of this rare syndrome follows. (Ann Hepatobiliary Pancreat Surg 2021;25:139-144)
\end{abstract}

Key Words: Cholecystoduodenal fistula; Gallstone ileus; Obstruction

\section{INTRODUCTION}

Bouveret syndrome is a rare variant of gallstone ileus and is defined as gastric outlet obstruction secondary to the impaction of a large gallstone in the pylorus or proximal duodenum. ${ }^{1}$ The obstruction occurs as a result of migration of the gallstone through a bilio-gastric or bilioenteric fistula into a lumen that cannot accommodate its size. Potential variants of causative fistulae include cholecystoduodenal (60\%), cholecystocolic (17\%), cholecystogastric (5\%), and choledochoduodenal (5\%). ${ }^{2,3}$ Gallstone ileus is a relatively rare entity accounting for only $1-4 \%$ of cases of small bowel obstruction. Bouveret syndrome is even more rare, accounting for a mere $1-3 \%$ of cases of gallstone ileus. ${ }^{1,2}$ Although both conditions can occur at any age, they are more prevalent in the elderly due to longstanding cholelithiasis. Typical clinical features may include nausea, vomiting, abdominal pain, hematemesis, weight loss, and anorexia. ${ }^{1,4}$ The non-specific and infrequent nature of these symptoms impart difficulty to the diagnosis of Bouveret syndrome. ${ }^{2}$ We present three cases of
Bouveret syndrome and the subsequent management.

\section{CASE}

\section{Case 1}

An 88-year-old woman with a history of dementia, endstage renal disease, hypertension, and chronic cholelithiasis presented with nausea and non-bloody emesis of twoday duration. She reported no abdominal pain, fever, melena, or hematochezia. The patient was hospitalized two years prior for a similar presentation, during which a CT scan revealed a large gallstone within the gallbladder (Fig. 1). At that time duodenopathy with a post-bulbar duodenal stricture was identified via EGD, the etiology of which was believed to be chronic cholelithiasis leading to inflammatory changes of the duodenum with resultant stricture formation. Pathological examination failed to demonstrate malignancy and testing for Helicobacter pylori infection was negative. The patient refused surgical intervention at that time and was treated conservatively with dietary modifications.

Received: June 17, 2020; Revised: July 20, 2020; Accepted: July 24, 2020

Corresponding author: Brianne Runyan

Department of General Surgery, The Jewish Hospital, 4777 E. Galbraith Road, Cincinnati, OH 45236, USA

Tel: +1-513-686-5392, Fax: +1-513-686-5394, E-mail: briannerunyan@gmail.com

Copyright (C) 2021 by The Korean Association of Hepato-Biliary-Pancreatic Surgery

This is an Open Access article distributed under the terms of the Creative Commons Attribution Non-Commercial License (http://creativecommons.org/ licenses/by-nc/4.0) which permits unrestricted non-commercial use, distribution, and reproduction in any medium, provided the original work is properly cited. Annals of Hepato-Biliary-Pancreatic Surgery • pISSN: 2508-5778 - elSSN: 2508-5859 


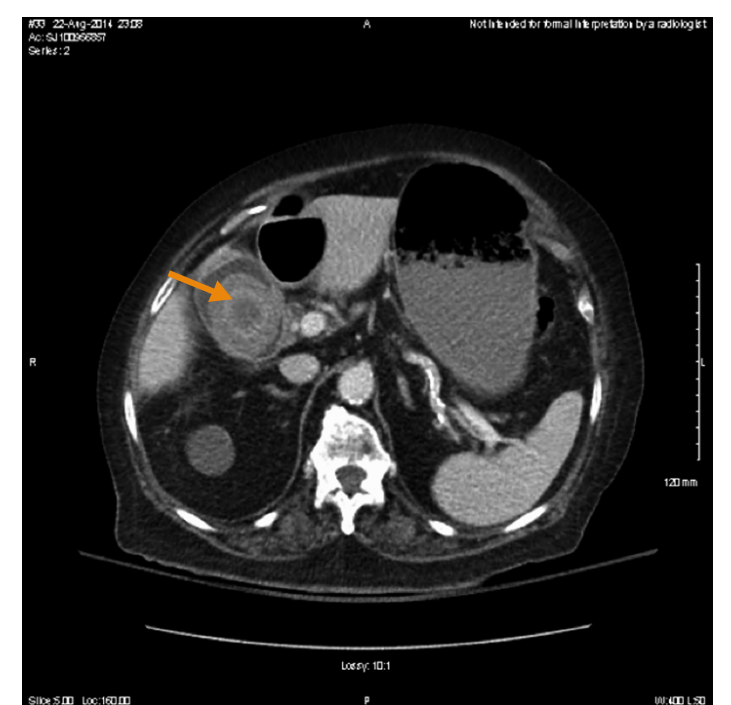

Fig. 1. Computed tomography images of an enlarging intraluminal gallstone. Axial view demonstrating interval enlargement of a gallstone within the gallbladder lumen. Arrow points to gallstone.

The patient presented two years later with moderate abdominal distention. On exam she was non-tender. Laboratory data were significant for an elevated white blood cell count of $16.7 \times 10^{9} / \mathrm{L}$ with a left shift and an alkaline phosphatase of 283 unit/L [25-100]. Her blood urea nitrogen was $11.8 \mathrm{mmol} / \mathrm{L}$ and creatinine was $556 \mu \mathrm{mol} / \mathrm{L}$, which was expected due to end-stage renal disease. Additional hepatic, hematologic and metabolic studies were unremarkable. An abdominal roentgengram revealed an abnormal gas pattern with a distended gastric silhouette and gastrectasis that was increased from prior studies. There was no pneumoperitoneum or significant calcifications in the right upper quadrant. The initial abdominal CT scan showed a large gallstone measuring $4 \mathrm{~cm}$ in the duodenum. A second abdominal CT scan was obtained five days later that showed progression of the large gallstone to the duodenal bulb with interval development of pneumobilia; there was no evidence of small bowel obstruction.

Initial management included nasogastric (NG) decompression followed by EGD, which revealed a normal appearing esophagus, gastric antrum and pylorus. A large gallstone measuring 4 to $5 \mathrm{~cm}$ was visualized at the apex of the duodenal bulb with surrounding mucosal edema. Several attempts were made to extract the stone endoscopically without success due to the angulation of the duodenum and the extensive inflammatory changes surrounding the stone.

Surgical intervention was pursued following failed en- doscopic extraction. The abdomen was entered laparoscopically and with hand-assistance. The gallstone in question was palpated; however, movement was difficult within the confines of the hand port. At this point, the procedure was converted to open. The lesser sac was entered and dissection was carried down to the duodenum. The gallstone failed to be manipulated proximally into the stomach secondary to dense adhesions. Despite meticulous lysis of adhesions and performing a limited Kocher maneuver, the attempts to move the stone into stomach were ultimately unsuccessful. Accordingly, the decision was made to pass the stone more distally in an attempt to extract it through a jejunal enterotomy. The stone moved freely down to the inferior and ascending portions of the duodenum; however, advancement beyond the ligament of Treitz was unsuccessful even after its release leading to the decision to proceed with a duodenotomy for stone extraction. The stone was manipulated back to descending portion of the duodenum and removed via duodenotomy on the antimesenteric border. The fistula was left intact and the duodenum was repaired primarily and covered with an omental patch. A feeding tube was left distally in anticipation of potential obstruction secondary to intramural hematoma. The patient was admitted for post-operative management. Tube feedings were started post-operative day 2 through the jejunostomy tube, while the gastrostomy tube remained to gravity. An upper gastrointestinal study was obtained on post-operative day 7 which showed no evidence of leak. The patient's diet was subsequently advanced as tolerated. She had return of gastrointestinal function on post-operative day 11 and was discharged home on post-operative day 14 . She is now over 24 months post-surgery without recurrence of symptoms.

\section{Case 2}

An 80 year old female with a history of hypertension, hypothyroidism, hyperlipidemia, and endometrial cancer status post pelvic radiation and hysterectomy, with previous abdominal surgery secondary to iatrogenic colonic perforation during colonoscopy, presented to an outside facility four days prior to arrival at our institution due to severe colicky epigastric, periumbilical, and right upper quadrant pain of one day duration associated with nausea and chills. Patient was made NPO with NG decompression in addition to receiving empiric meropenem at the outside facility where a cholecystoduodenal fistula with 
large gallstone in the duodenal lumen was identified by abdominal CT. Upper GI was performed on hospital day four at the outside facility, which revealed failure of stone progression prompting transfer to our institution for definitive management. On intake exam her vitals were stable within normal limits and she was found to have no abdominal tenderness and no distention. Her laboratory values were without any significant abnormalities.

Gastroenterology attempted endoscopic retrieval of the duodenal gallstone, at which time the stone was noted to be $3.5 \mathrm{~cm}$ in diameter and obstructing the lumen of the bulbar duodenum. Endoscopic retrieval attempts resulted in partial fragmentation of the stone but were ultimately unsuccessful. The following day repeat abdominal CT revealed migration of the stone into the proximal jejunum (Fig. 2).

The decision to proceed with surgical removal hinged on the concern that further distal transit of the stone may result in it becoming lodged in a bowel loop contained in the patient's incisional hernia or a pelvic loop due to previous pelvic radiation. The abdomen was entered laparoscopically. Multiple areas of adhesions were encountered requiring adhesiolysis and ultimately the insertion of a hand port. The stone-containing small bowel was located and delivered through the hand port after which two stay sutures were placed on the antimesenteric border. An en-

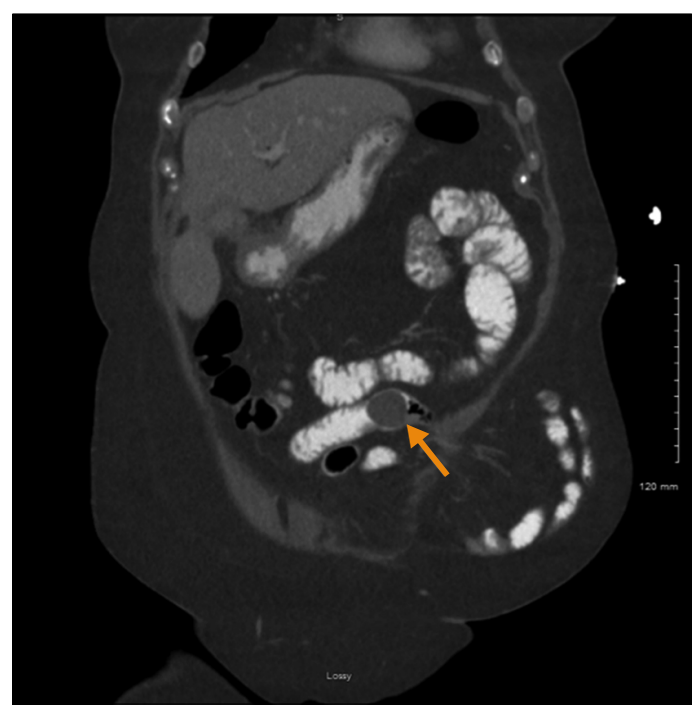

Fig. 2. Computed tomography images showing jejunal gallstone. Coronal view demonstrating distal migration of the gallstone from the duodenum to the jejunum. Arrow points to gallstone. terotomy was then made through which the gallstone was subsequently delivered (Fig. 3). Closure of the enterotomy was achieved via two transverse layers of suture and the bowel was returned into the abdomen. The cholecystoduodenal fistula was left intact for closure at a later date. The patient was transferred back to the general medical/surgical floor for post-operative recovery. Her diet was advanced on post-operative day 1 and she experienced return of gastrointestinal function on post operative day 2. The patient was discharged home on post operative day 2. She is now 16 months post-surgery and has had no recurrent symptoms.

\section{Case 3}

A 68 year old female with history of sarcoma, non-small cell lung cancer, breast cancer, diabetes, nephrolithiasis, hyperlipidemia, and surgical history of hysterectomy approximately one year prior to presentation, presented with intermittent periumbilical abdominal pain of one day duration. She reported multiple episodes of non-bloody non-bilious emesis with subsequent but transient resolution of the presenting pain. The patient reported a recent 25 pound unintentional weight loss. On exam she was afebrile and hemodynamically stable with mild tachycardia. Her abdominal exam was significant for periumbilical guarding and right upper quadrant tenderness. Laboratory values revealed an elevated lactic acid as well as a leukocytosis. All

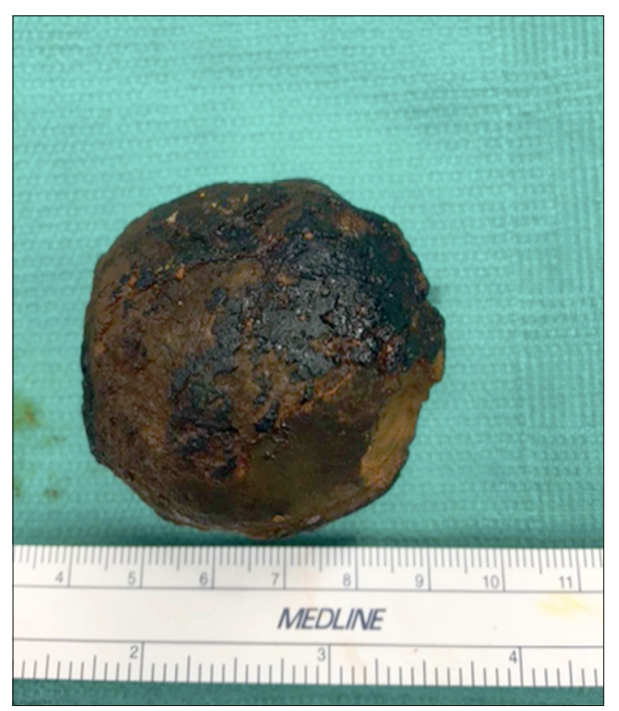

Fig. 3. Gross pathologic specimen from surgery. This image demonstrates the gross pathology of a large gallstone measuring $5 \mathrm{~cm}$. 

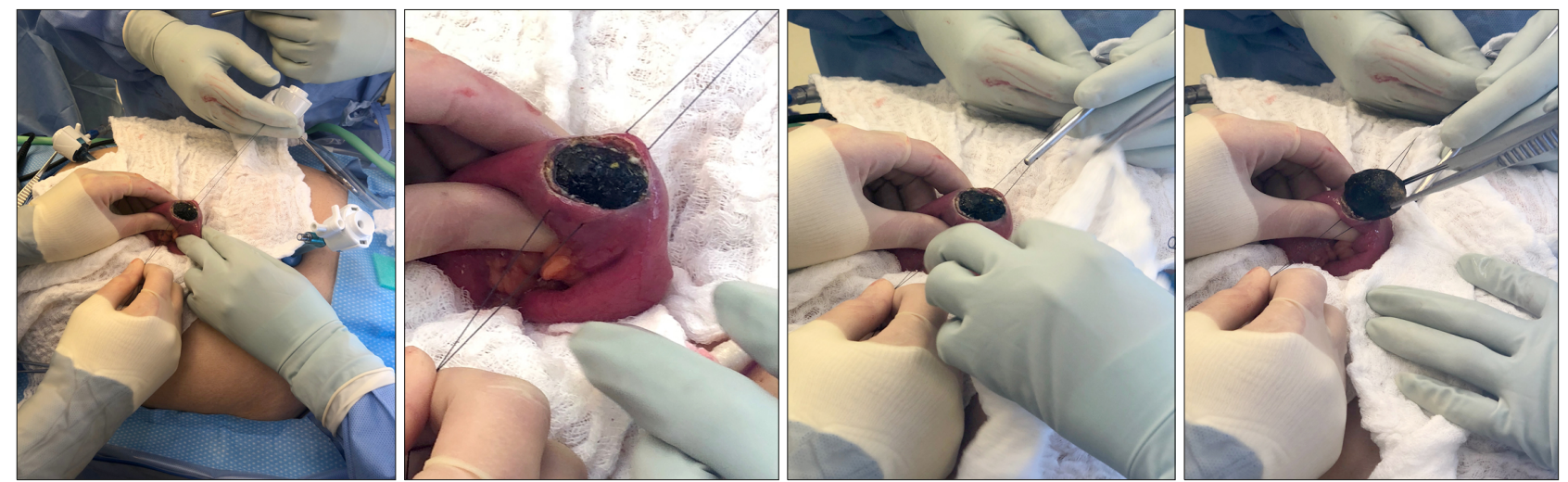

Fig. 4. Gross view of enterotomy created for stone extraction.

other laboratory values were without significant abnormalities. An abdominal CT scan revealed extensive inflammation of the gallbladder fossa along with a choledochoenteric fistula and gallstone within the jejunum.

The decision was made to proceed to the operating room for diagnostic laparoscopy with possible small bowel resection. The abdomen was entered laparoscopically. Multiple areas of adhesions were encountered requiring adhesiolysis. A hand port was later added secondary to the severe extent of adhesions. The gallstone-containing loop of bowel was identified and delivered through the hand port. Two stay sutures were placed, an enterotomy was created, and the gallstone was delivered (Fig. 4). The enterotomy was then closed with two layers of transverse sutures and the bowel loop was returned into the abdominal cavity. The fistula was left intact to be addressed at a later date. The patient was transferred to the general medical/surgical floor for post-operative recovery. She experienced respiratory insufficiency post-operatively requiring high flow supplemental oxygen; her NG remained in place until post-operative day 2. Bowel function returned by post-operative day 3 and she tolerated advancement of diet without any issues. The patient remained hospitalized until post-operative day 5 in an attempt to improve her respiratory insufficiency with aggressive pulmonary toilet; however, she was ultimately discharged with home oxygen, which she had previously required earlier in the year. The patient expired nine months later due to an unrelated etiology; she did not experience obstruction or cholecystic symptoms from the time of operative management until her unrelated death.

\section{DISCUSSION}

Bouveret syndrome is named after Leon Bouveret, who published two case studies of this condition in $1896 .{ }^{5}$ It is defined as gastric outlet obstruction secondary to the impaction of a large gallstone in the distal stomach, pylorus or proximal duodenum. The obstruction occurs as a result of the migration of a gallstone through a bilio-gastric bilio-enteric fistula., ${ }^{2,3}$ Bouveret syndrome is a sequela of chronic cholelithiasis, which leads to inflammation, ischemia and necrosis of the gallbladder wall with subsequent erosion into the duodenum, a progression which helps to explain the observation that elderly patients tend to develop Bouveret syndrome more frequently than younger patients. ${ }^{2}$ According to Cappell and Davis, ${ }^{4}$ who reviewed all of the published reports of Bouveret syndrome since 1974 , there is a female predominance (female-to-male ratio of 1.86) and a mean age at diagnosis of 74.1 years ( $\mathrm{SD}=11$ years). These trends hold true for all three of our cases presented in this series.

The typical presentation of Bouveret syndrome involves nausea/vomiting $(86 \%)$ and abdominal pain or discomfort (71\%). Less common symptoms include hematemesis (15\%), anorexia $(16 \%)$ and early satiety $(3 \%){ }^{4}$ The presence of these symptoms can be variable depending on the degree of inflammation and edema in the gastric tissue surrounding the stone. Further, the non-specific nature of these gastrointestinal symptoms lends challenge to arriving at this rare diagnosis. The patients discussed herein presented with abdominal pain and emesis secondary to gastric outlet obstruction, which is consistent with the most common presentation. Abdominal x-ray has historically 
been cited as the imagining modality most used for diagnosis which had been observed to be diagnostic in onefifth of cases, typically revealing calcification in the right upper quadrant. ${ }^{4}$ In modern times, however, abdominal CT has assumed the gold standard role for diagnosis and typically reveals Rigler's triad of pneumobilia, an ectopic gallstone and gallstone ileus. ${ }^{2,6}$ All three of the cases presented in this series were diagnosed by CT demonstration of Rigler's triad features.

Management of Bouveret syndrome typically begins with endoscopy, which can be both diagnostic and therapeutic. However, EGD has been shown to be diagnostic in only $69 \%$ of cases, the remainder of which gallstones may be left undiscovered for reasons such as being deeply embedded within the gastric or enteric mucosa or may appear as an obvious hard mass that is convex, smooth, non-friable and non-fleshy. ${ }^{4}$ Despite endoscopic retrieval being the typical first choice for management, only about $10 \%$ of attempts were found to result in successful retrieval. Two of the three cases in this series followed the standard treatment algorithm, both of which were successful from a diagnostic standpoint but unsuccessful with regard to stone extraction.

Stones that cannot be retrieved endoscopically require surgical intervention. The most common surgical approach includes manipulating the stone proximally into the stomach and removing it via a gastrotomy. The stone can also be directed distally to the jejunum and an enterotomy performed to facilitate gallstone extraction. Duodenotomy is not the preferred approach because of the retroperitoneal orientation of the majority of the duodenum; however, in one of our cases it was necessary due to the inability to manipulate the gallstone into either the stomach or jejunum for extraction. In that case we demonstrated that duodenotomy is a safe technique for difficult gallstone extraction when performed selectively. Cholecystectomy and fistula closure are not an absolute requirement because the fistula may spontaneously close if the cystic duct is patent and residual gallstones are not present. ${ }^{1,2,4,6}$ None of the cases presented in this series involved immediate cholecystectomy or fistula closure and none of the patient's developed recurrent symptoms.

While the literature mainly discusses endoscopic and open surgical removal of the stone, the majority of endoscopic attempts are unsuccessful and an open gastrotomy, pylo- rotomy, or duodenotomy needs to be done. Given the patient population that Bouveret syndrome affects, any open procedure is associated with significant morbidity and mortality. ${ }^{7}$ Advances in technology bring into question the use of laparoscopic surgery as an acceptable option. Laparoscopic enterolithotomy has been reported as safe and effective in the management of Bouveret syndrome. Described techniques include stone removal through a laparoscopic duodentomy or pyloroduodenotomy with a transverse closure. ${ }^{7}$ Malvaux et al. $^{8}$ described laparoscopically manipulating the gallstone into the stomach, fragmenting it and then extracting it through a gastrotomy. As with the open technique, is important to examine the remainder of the small bowel for other stones to prevent any future obstructions. If laparoscopic expertise and equipment are available and endoscopic therapies have failed, laparoscopic removal of the stone should be the preferred treatment. ${ }^{7}$

Bouveret syndrome is a rare clinical entity that involves a bilio-gastric or bilio-enteric fistula that carries a mortality rate of approximately $12 \%$. This mortality is often attributed to the advanced age and co-morbidities of the patients most commonly affected. Surgeons should be vigilant when approaching elderly patients with a small bowel obstruction or gastric outlet obstruction, especially when a biliary fistula is suspected. While relatively uncommon and often overlooked, Bouveret syndrome should be included in the list of differential diagnoses when considering small bowel obstruction. In addition, if patients are found to have this rare entity, a discussion with the patient should be partaken about the importance of early intervention. A delay in diagnosis and treatment may lead to unnecessary morbidity and mortality in at-risk populations. The cases reported in this series all required surgical management of Bouveret syndrome with resultant favorable long-term outcomes.

\section{ACKNOWLEDGEMENTS}

Thank you to our families, friends and colleagues who support us in our work. Supported by The Jewish Hospital, General Surgery, Cincinnati, OH.

\section{CONFLICT OF INTEREST}

The authors declare that they have no conflicts of in- 
terest.

\section{INFORMED CONSENT STATEMENT}

Informed consent was waived.

\section{ORCID}

Brianne Runyan: https://orcid.org/0000-0002-4026-4778

Michael L. Caparelli: https://orcid.org/0000-0003-3451-538X

Jason Batey: https://orcid.org/0000-0003-3196-9255

Shyam Allamaneni: https://orcid.org/0000-0002-2779-3296

Steven Perlman: https://orcid.org/0000-0002-9980-9816

\section{AUTHOR CONTRIBUTIONS}

Contributed to the design, implementation, data collection, interpretation and drafting of this manuscript: BR, MLC, JB, SA. Contributed to the design, interpretation, radiologic imaging and drafting of this manuscript: SP.

\section{REFERENCES}

1. O’Neill C, Colquhoun P, Schlachta CM, Etemad-Rezai R, Jayaraman S. Gastric outlet obstruction secondary to biliary calculi: 2 cases of Bouveret syndrome. Can J Surg 2009;52:E16E18.

2. Iancu C, Bodea R, Al Hajjar N, Todea-Iancu D, Bălă O, Acalovschi I. Bouveret syndrome associated with acute gangrenous cholecystitis. J Gastrointestin Liver Dis 2008;17:87-90.

3. Masannat YA, Caplin S, Brown T. A rare complication of a common disease: Bouveret syndrome, a case report. World J Gastroenterol 2006;12:2620-2621.

4. Cappell MS, Davis M. Characterization of Bouveret's syndrome: a comprehensive review of 128 cases. Am J Gastroenterol 2006; 101:2139-2146.

5. Ramos GP, Chiang NE. Bouveret's syndrome. N Engl J Med 2018;378:1335.

6. Brennan GB, Rosenberg RD, Arora S. Bouveret syndrome. Radiographics 2004;24:1171-1175.

7. Caldwell KM, Lee SJ, Leggett PL, Bajwa KS, Mehta SS, Shah SK. Bouveret syndrome: current management strategies. Clin Exp Gastroenterol 2018;11:69-75.

8. Malvaux P, Degolla R, De Saint-Hubert M, Farchakh E, Hauters P. Laparoscopic treatment of a gastric outlet obstruction caused by a gallstone (Bouveret's syndrome). Surg Endosc 2002;16:11081109. 